\title{
Maple syrup urine disease hair reveals the importance of 18-methyleicosanoic acid in cuticular delamination
}

\author{
James R. Smith ${ }^{\mathrm{a}, *}$, J. Alan Swift ${ }^{\mathrm{b}}$

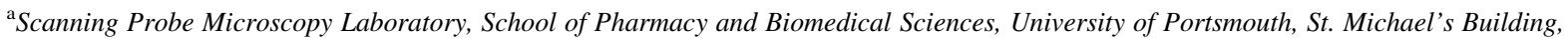 \\ White Swan Road, Portsmouth PO1 2DT, UK \\ ${ }^{\mathrm{b}}$ Department of Human Anatomy and Cell Biology, University of Liverpool, Sherrington Buildings, Ashton Street, Liverpool L69 3GE, UK
}

Received 3 August 2004; revised 12 November 2004; accepted 15 November 2004

\begin{abstract}
18-Methyleicosanoic acid (18-MEA) is thought to be covalently bound to the outer surface of human hair and is a major component of the outer $\beta$-layer of the cuticular cell membrane complex (CCMC). Cuticular delamination, whether this occurs between the outer $\beta$ - and $\delta$ layers or within the plane of the $\beta$-layer, results in a fresh layer of 18-MEA being exposed at the newly-revealed surface. Hair from patients with Maple Syrup Urine Disease (MSUD), however, does not contain 18-MEA and here, we report on the importance this unusual fatty acid in cuticular delamination. Hair fibres were collected from 10 patients with classic (type 1A) MSUD from a Mennonite community in Pennsylvania, USA. Included amongst these were hairs from dizygotic twins (A1 and A2), one of whom had MSUD, and the other did not; it was unknown at the beginning of the study which twin had MSUD. The outer surfaces were examined using atomic force microscopy (AFM) and transverse sections imaged using transmission electron microscopy (TEM). The newly revealed intercellular surface regions from twin A2 were found to be significantly rougher than those from twin A1. TEM studies showed the trilamellar CCMC to be continuous for twin A1, but possessed discontinuities of variable length (100-1000 nm) for twin A2. In contrast with other work, TEM showed no specific defects in the outer $\beta$-layer. The outer cuticular surfaces for most MSUD patients showed a great abundance of residual endocuticle, although in other cases this was less pronounced. These differences may be explained by some residual activity of the branched-chain $\alpha$-ketoacid (BCKD) dehydrogenase. Cuticular delamination in MSUD-hair probably still occurs within the general plane of the CCMC, although fracture through discontinuities of this layer results in zones of endocuticle being exposed at the new surface.
\end{abstract}

(C) 2005 Elsevier Ltd. All rights reserved.

Keywords: 18-Methyleicosanoic acid (18-MEA); Atomic force microscopy (AFM); Cuticle; Maple syrup urine disease (MSUD); Hair; Transmission electron microscopy (TEM)

\section{Introduction}

The discovery of fatty acids found covalently-linked to the surfaces of keratin fibres (Evans et al., 1985; Wertz and Downing, 1988, 1989) has led to much renewed interest in the surface chemistry and properties of these materials (Jones and Rivett, 1997; Swift, 1999). 18-Methyleicosanoic acid (18-MEA), an unusual, branched-chain fatty acid, is a major component of the outer $\beta$-layer of the cuticular cell membrane complex (CCMC), located on the underside of the $\delta$-layer. The layer of 18-MEA is thought to be covalently

\footnotetext{
* Corresponding author.

E-mail address: james.smith@port.ac.uk (J.R. Smith).
}

bound, probably via a thioester or ester linkage, to the underlying proteinacous epiciticle (Evans and Lanczki, 1997) (Fig. 1). Interestingly, the inner $\beta$-layer, of the CCMC, does not contain 18-MEA and is thought to be comprised mainly of the straight-chain fatty acids, palmitic (C16:0) and oleic (C18:0) acids (Jones and Rivett, 1997). The precise role of 18-MEA is uncertain (Jones and Rivett, 1997), although the large segmental volume of the anteisoterminus is expected to exhibit considerable molecular mobility, resulting in fluid-like behaviour at cuticle surfaces (Swift, 1999) and a possible explanation for the observed differential friction effect (DFE) of hair (Swift, 1999; Adams et al., 1990). These properties have been described as being important for hair detanglement, alignment 


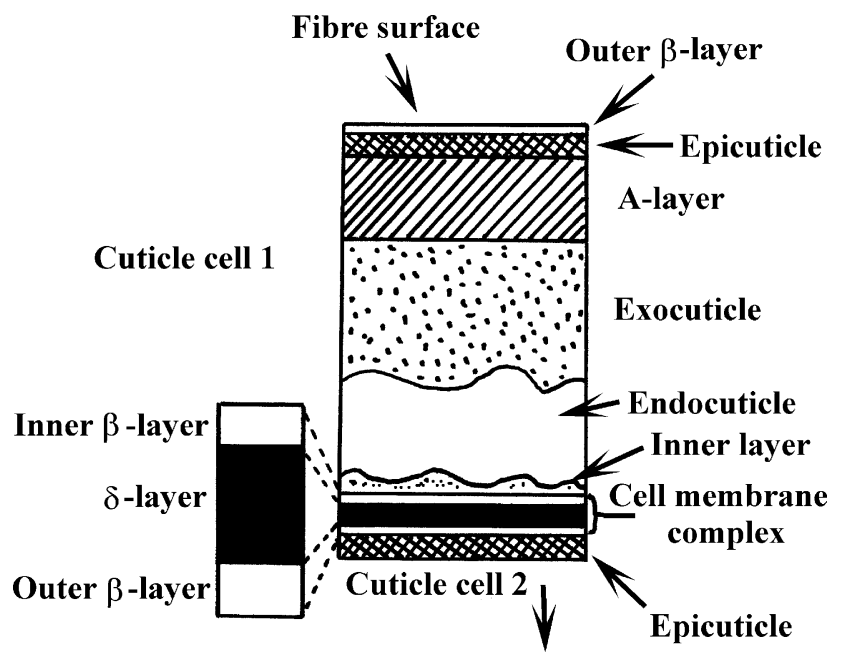

Fig. 1. Schematic diagram of the lamellar sub-structure of the human hair cuticle. (Reproduced with permission (Swift and Smith, 2001)).

and rejection of scalp detritus (Martin, 1944) and are of obvious commercial importance to the hair toiletries industry.

Whether the $\beta$-layer is of single lipid layer thickness or is present as a lipid bilayer (Swift and Holmes, 1965) is uncertain. Either (a) there is only one layer of lipid covalently attached to the adjacent cell with it is outer region abutting the $\delta$-layer, or (b) there are two layers of intercalated lipid with one layer covalently attached to the adjacent cell and the other (covalently) attached to the $\delta$-layer. A weak point for cuticular delamination is therefore expected at the CCMC, either at the interface between the outer $\beta$ - and $\delta$-layers (single lipid model) or within the outer $\beta$-layer (lipid bilayer model). Regardless of the cleavage pathway, 18-MEA provides at least one side of the fracture plane and it is this fatty acid that will be exposed at the newly revealed fibre surface.

The absence of 18-MEA from hairs of patients with Maple Syrup Urine Disease (MSUD) provides an interesting route to investigate the importance of this anteiso, branchedchain fatty acid (Jones et al., 1996). MSUD, first described by Menkes et al. in 1954 (Menkes et al., 1954), is an inherited metabolic and progressive neurological degenerative disorder where decarboxylation of branched-chain $\alpha$ ketoacids derived from leucine, isoleucine and valine is blocked (Dancis et al., 1960). The accumulation of these branched chain fatty acids to toxic levels will result if not controlled through special dietary arrangement (Chuang, 1998). MSUD is caused through various mutations that can occur in the E1 $\alpha, \mathrm{E} 1 \beta, \mathrm{E} 2$ and E3 loci of the mitochondrial branched-chain $\alpha$-ketoacid (BCKD) dehydrogenase complex (Chuang, 1998). The disorder has five distinct phenotypes (Chuang and Shih, 1995), of which the classic type, first described by Menkes (Menkes et al., 1954), is the most severe with the lowest residual activity of $0-2 \%$ of normal (Chuang, 1998). Higher residual activities, 3-30\%, are found for the milder intermediate and intermittent types of the disorder (Dancis et al., 1972).

Although MSUD is a rare autosomal disease (1 in 185 000) (Silao et al., 2004), in the Mennonite communities of Pennsylvania, USA, the occurrence is 1 in 176 of live consanguineous births (DiGeorge et al., 1982; Fisher et al., 1991; Matsuda et al., 1990). Mennonite MSUD is of the classic type (type 1A), where a specific mutation, a Y393N substitution, occurs in the E1 $\alpha$ loci causing degradation of the E1 $\beta$ subunit (Fisher et al., 1991; Matsuda et al., 1990).

Mutations in the BCKD enzyme result in the inability of 18-MEA to be biosynthesised from its precursor, isoleucine (Jones et al., 1996). In MSUD hair, 18-MEA is replaced by straight-chain fatty acids, such as n-eicosanoic acid (15-19\%) (Jones et al., 1996). High-resolution transmission electron microscopy (TEM) studies of transverse-sections of hair obtained from MSUD patients have show a structural defect in the outer $\beta$-layer that have led to the conclusion 18MEA was closely associated with this layer (Jones et al., 1996).

In this study, hair fibres were collected from 10 patients with classic (type 1A) MSUD from a Mennonite community in Pennsylvania, USA. The outer surfaces were examined using atomic force microscopy (AFM) (Smith, 1998; Smith and Swift, 2002; Swift and Smith, 2000, 2001) and transverse sections imaged using TEM to investigate the importance of 18-MEA in cuticular delamination.

\section{Experimental}

Small tresses of scalp hairs from 10 classic-MSUD patients (neonates and adults) were obtained from the Clinic For Special Children, Strasburg, PA, USA. Included among these were hairs from dizygotic twins (A1 and A2) where one twin had MSUD and the other did not. Not until the completion of the work was it revealed which twin had MSUD. Ten hairs from each of the 10 subjects were placed in an aqueous solution of sodium dodecylsulphate (1\%) and agitated in an ultrasonic bath for $2 \mathrm{~min}$, rinsed extensively with double-distilled water and dried in a gentle stream of nitrogen. This procedure in known to be very effective in removing detritus from hair surfaces (Swift, 1991).

For TEM studies, hairs from the dizygotic twins (A1 and A2) were embedded without pre-treatment in size 0 gelatin capsules using Spurr's resin (Spurr, 1969). Transverse sections of ca. $60 \mathrm{~nm}$ thickness were cut on a diamond knife using a Reichert-Jung 'Ultracut' ultramicrotome and collected on 400-mesh gold electron microscope grids previously coated with a thin collodion supporting membrane. The grids were stained by immersing for $2 \mathrm{~h}$ at room temperature in a saturated solution of phosphotungstic acid (PTA) in 50\% ethanol. These were rinsed for $5 \mathrm{~s}$ in $50 \%$ ethanol and then rapidly drained to dryness on the edge of a filter paper. The grids were examined in a ZEISS EM109 transmission electron microscope operated at $50 \mathrm{keV}$ 
and images were recorded on black-and-white roll film (Agfa Ortho 25).

Topographic AFM studies were performed using a Discoverer TMX2000 Scanning Probe Microscope (SPM) (Veeco Instruments, Cambridge, UK). Imaging was performed in contact mode using standard-profile, silicon nitride tips mounted on $\mathrm{V}$-shaped cantilevers of length and nominal force constant, $k, 200 \mu \mathrm{m}$ and $0.032 \mathrm{~N} \mathrm{~m}^{-1}$, respectively (Veeco Instruments, Cambridge, UK). A piezo-electric tripod scanner with a maximum $x, y, z$ translation of $70 \times 70 \times 12 \mu \mathrm{m}$ was used. For examination of hair surfaces, ten images were randomly selected along the length of each cut segment; the scan-range was limited to below $20 \times 20 \mu \mathrm{m}$ owing to the gross curvature of hair surfaces. Representative AFM images were left-shaded using a hypothetical light source to enhance topographic features.

\section{Results and discussion}

Typical AFM images representative of scalp hairs taken from dizygotic twins, A1 and A2, respectively, are shown in Fig. 2(a) and (b). Although the distance from the scalp was not known, the presence of false scale edges (marked ' $G$ ' in Fig. 2) and longitudinal striations (Swift and Smith, 2000) provided an approximate indication as to the region along the length each image was acquired. The fine cuticular surface architecture of hair observed from A1 appeared to be very similar to that exhibited in normal hair and very little residual endocuticle was observed in the micrographs. In contrast, almost all of the images obtained from the hair cuticle of twin A2 showed that the newly revealed intercellular surface regions (marked ' $N$ ') were significantly rougher than those of twin A1 (Fig. 3) and those observed in normal hair. The roughening of $\mathrm{N}$ at regions close to the root-end $(<2 \mathrm{~cm})$, as evidenced by the highly striated outer surfaces, leads to the conclusion that there must be some underlying physioloigical cause rather than being due to erosion. Closer examination of the roughened regions revealed the presence of step-like irregularities that have arisen from uneven delamination through the sublamellar structure of the CCMC.

TEM examination of the four hairs examined from twin A1 revealed the internal structure to be perfectly normal. In particular, the CCMC separating the cuticle cells from each other was continuous and exhibited the usual sharply defined structure of two thin and unstained $\beta$-layers either side of a heavily stained $\delta$-layer (Fig. 4(a)). The same trilamellar structure of the CCMC was also present in the four hairs examined from twin A2, with similar thicknesses of all three layers as for twin A1. However, in these hairs there was significant lack of continuity of the CCMC between adjacent cells. They generally took the form of localised pervasions by endocuticle of the region where the CCMC would normally be located resulting in a welldefined slightly irregular boundary with the epicuticle of the cell beneath (Fig. 4(b)). These regions of CCMC discontinuity were of variable length, for the most part extending a distance of between 100 and $1000 \mathrm{~nm}$. Occasionally, they extended for as much a $1 \mu \mathrm{m}$ (Fig. 4(c)), giving the clear impression of the contents of adjacent cuticle cells being fused together without the intervention of a CCMC.

Typical AFM images of the outer surfaces of hairs obtained from four MSUD patients are shown in Fig. 5. The cuticular architecture of patient B showed appreciable distal scale breakage at distances relatively close to the scalp, as evidenced from the presence of striations which are only observed on the original outer cuticular sheets (Fig. 5(a) and (b)). Breakage at the sides of cuticular scales is also apparent in both micrographs, a feature not commonly observed in normal hair. Significant distal breakage at regions close to the scalp was characteristic of all the MSUD hairs investigated. Similar to the hair outer surfaces of MSUD twin A2, roughened cuticular sheets were also observed for Patient C (Fig. 5(c)). Some deposits of residual endocuticle were also found at the foot of the scale edges for this patient, although the extents of these were not too uncommon from those observed with normal hair. In contrast, however, a great abundance of residual endocuticle was apparent on the outer cuticle surfaces from Patient D (Fig. 5(e)-(h)); this extended for distances significantly
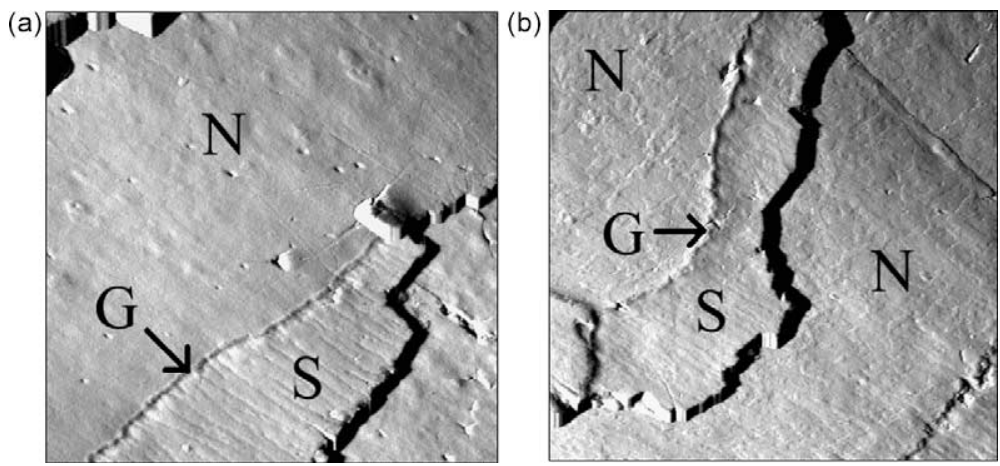

Fig. 2. AFM images of the outer surface cuticle of hair from twins (a) A1 and (b) A2. N: Newly revealed surface region; G: scale-edge ghost (5); S: striated original outer surface of the fibre. $x=y=10 \mu \mathrm{m}$. Cuticle scales overlap from top-left (nearer to root end) to bottom-right. 


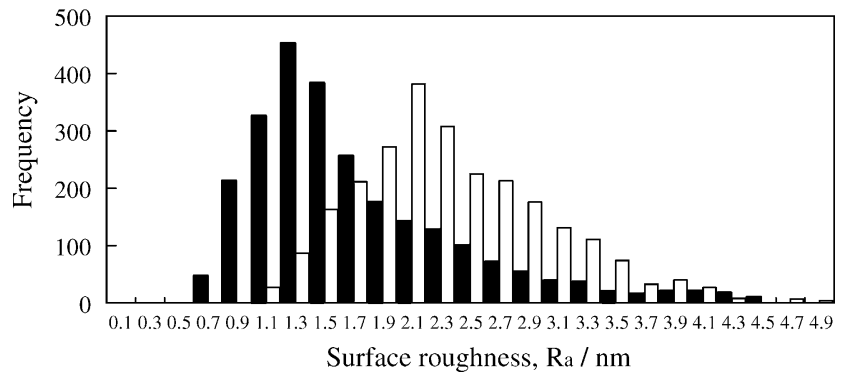

Fig. 3. Surface roughness histograms of the newly revealed surface regions (N) of hair for twins A1 (black bar) and A2 (white bar). The frequency axis refers to the number of incidences of the surface roughness band.

forward from the scale edges and is not observed in normal hair. In some regions, large 'holes' in cuticle surfaces were seen (e.g. Fig. 5(e)). These may be due to breakages across the discontinuities in the CCMC (as in Fig. 4(c)), revealing fused endocuticle (Fig. 6). Patient E showed some distal cuticle breakage (Fig. 5(i)), but as with Patient C (Fig. 5(c)), this was fairly typical of that observed in non-MSUD hair.

Our thesis is that mechanical wear at the surface of normal hair results in preferential delamination in the CCMC close to the distal edges of the scales at the interface between the outer $\beta$-layer and the $\delta$-layer (Swift and Smith, 2000) or within the plane of the outer $\beta$-layer (Fig. 6). As small pieces chip away from the scale margins, a fresh surface of outer $\beta$-layer is revealed that is seen by AFM to be relatively smooth. Whilst in MSUD-hair delamination probably still occurs within the general plane of the CCMC, fracture through the discontinuities of this layer result in zones of granular-structured endocuticle being exposed at the new surface (Fig. 6). It therefore seems that 18-MEA is essential for maintaining the structural integrity and continuity of the CCMC and the separation of the cuticle cells from each other in normal hairs. It is not clear whether the CCMC discontinuities seen in MSUD hair arise simply because of insufficient 18-MEA for the complete CCMC to be formed or whether they arise in the follicle after other alternative fatty acids have been incorporated into a continuous CCMC (such as C18:0 or C20:0, cf. Kao Corporation, 1991). One possibility is that in normal hair, 18-MEA ensures smoothness of intercellular sliding of the early cuticle cells in the follicle, i.e. during the 'zipper-like' flattening and tilting process that yields the characteristic system of overlapping cuticle cells (Mercer, 1961). A greater level of cohesivity, as might occur in MUSD if straight-chain rather than anteiso-fatty acids are incorporated into the CCMC, could well lead to localised rupture of the CCMC during this cellular realignment process.

Whilst an abundance of residual endocuticle was observed on some MSUD hairs, other fibres were fairly clean showing only small deposits as seen in non-MSUD hair. A reason for this may reflect physiological differences in the MSUD condition. For example, clean surfaces relatively free from granular endocuticle may result when cuticular delamination occurs predominantly through the CCMC owing to sufficient residual enzyme activity that could biosynthesise some 18-MEA. If the BCKD enzyme is significantly mutated, however, large amounts of endocuticle deposits will still be observed on the outer hair surfaces even if MSUD patients have returned to good metabolic
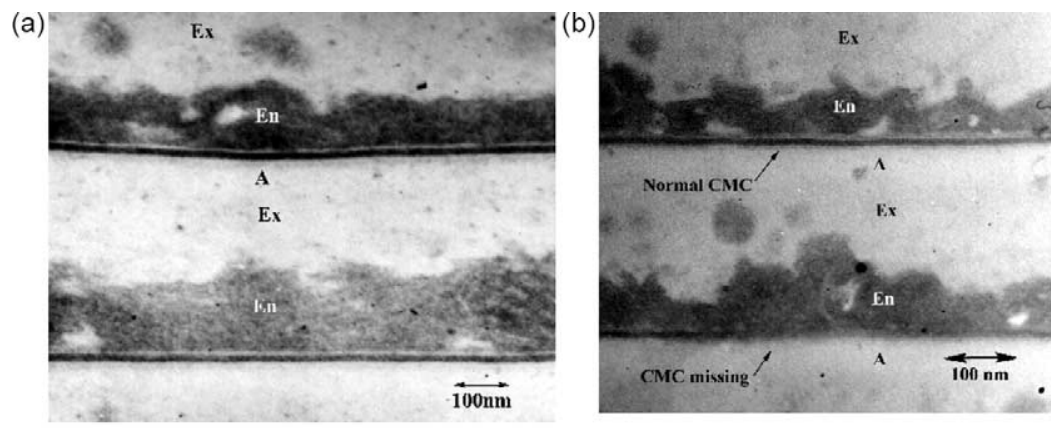

(c)

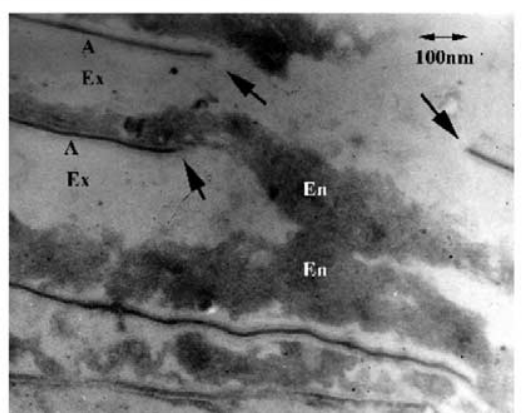

Fig. 4. TEM transverse sections of hair from twins A1 and A2. (a) A1 shows normal cuticle structure; (b,c) A2 shows abnormal cuticle structure. The CCMC between some cuticle cells is normal (b), but in other cases (lower arrow) the CCMC is absent for a short distance. In (c), the CCMC is grossly discontinuous (arrowed) and the contents of adjacent cells are fused by the mixing of endocuticle. A, A-layer; Ex, exocuticle; En, endocuticle. 

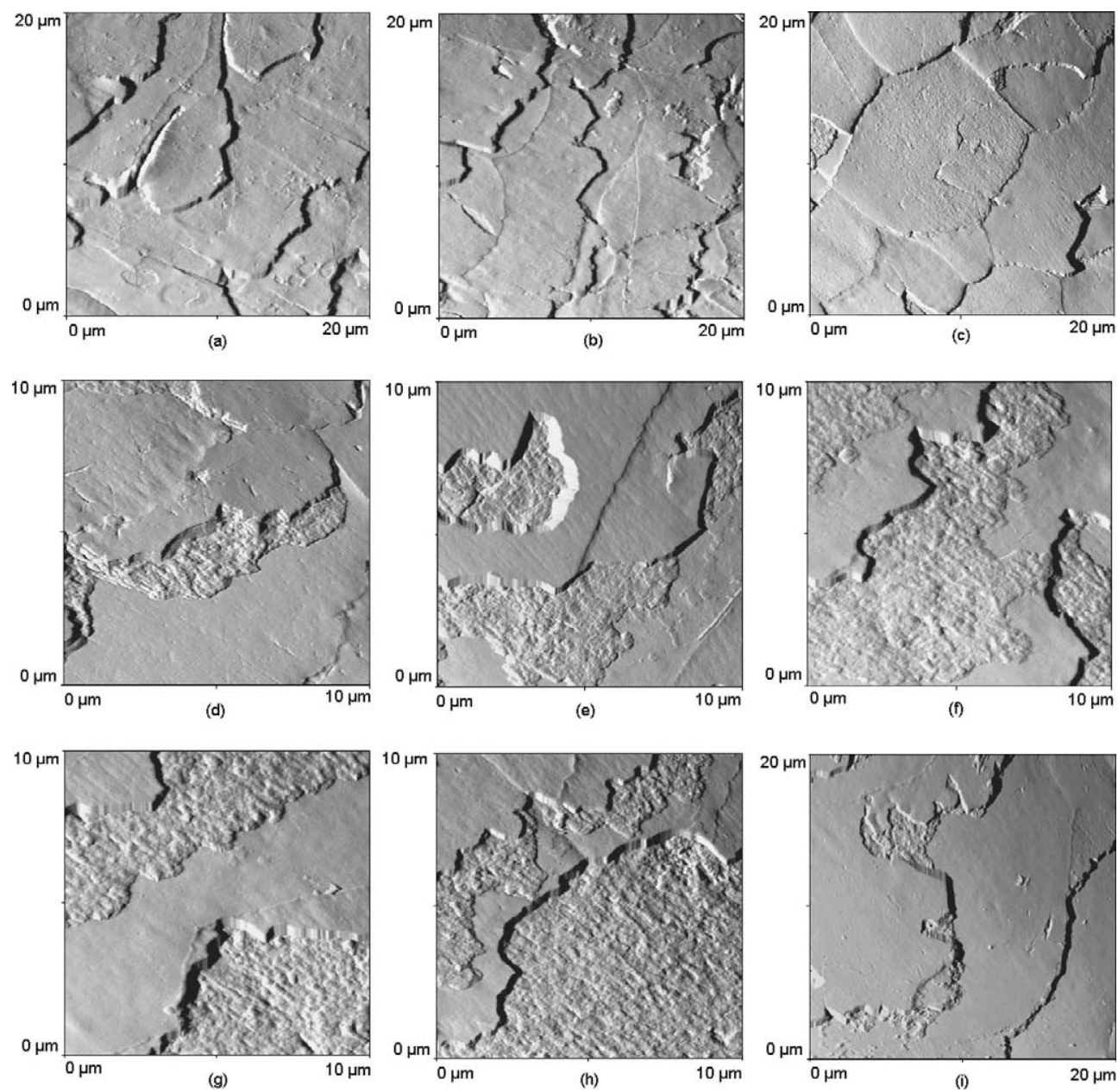

Fig. 5. Typical AFM images of the outer surfaces of human hairs obtained from four patients with MSUD: (a,b) Patient B, (c,d) patient C, (e-h) patient D, (i) patient E. Cuticle scales overlap from top-left (nearer to root end) to bottom-right.

control through appropriate dietary intake. Microscocopic evaluation of MSUD hair fibres, therefore, does not show promise for use as a diagnostic tool for the control of the disease, but it does reveal the importance of 18-MEA in cuticular delamination and may highlight physiological differences in the disease.

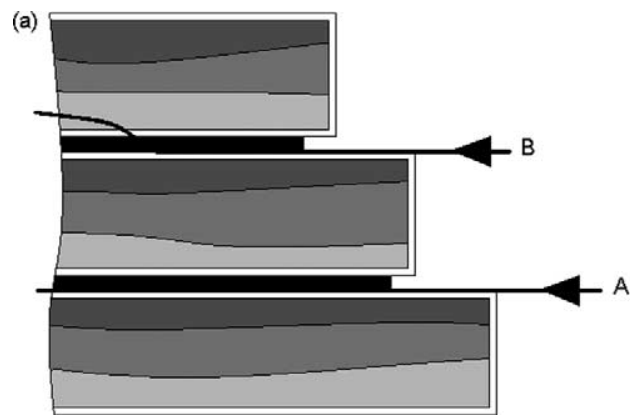

\section{Conclusion}

AFM and TEM examinations of the outer surfaces and transverse sections of hair surfaces from MSUD patients suggests that 18-MEA is essential for maintaining the structural integrity and continuity of the CCMC and

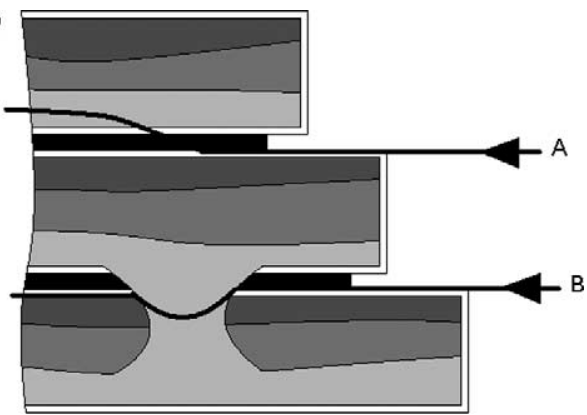

Fig. 6. Mechanisms for cuticular delamination in (a) non-MSUD and (b) MSUD hair before vertically tearing upwards through the cuticle: (a) cleavage occurs between the outer $\beta$ - and $\delta$-layers (or within the outer $\beta$-layer) (A) with some fracture through the endocuticle (B); (b) cleavage similar to (a)(A), but more fracture through the endocuticle (A) and fracture through soft bridging endocuticle resulting in 'holes' in newly-revealed cuticle surfaces exposing endocuticle (B). Dark grey $=$ A-layer, mid-grey $=$ exocuticle, light-grey $=$ endocuticle, black $=\delta$-layer of the CCMC, white $=\beta$-layers. 
the separation of the cuticle cells from each other in normal hair.

AFM revealed the surfaces of many of the MSUD hairs to be very heavily covered in a granular deposit of endocuticle. This is thought to be due to some degree of fracture through this layer as the propagation encounters a defect within the plane of the CCMC. The variable extent of granular endocuticle coverage on outer surfaces may reflect physiological differences relating to the residual BCKD activity.

\section{Acknowledgements}

We thank Dr D.H. Morton of the Clinic For Special Children, Strasburg, PA, USA for the generous provision of MSUD hair samples and the Royal Society of Chemistry for financial support. We also thank Deutsches Wollforschungsinstitut, Aachen, Germany and particularly Professor F.-J. Wortman, for providing the facilities for the TEM work. We thank Wella AG of Darmstadt for funding the TEM work.

\section{References}

Adams, M.J., Briscoe, B.J., Lee, T.K., 1990. The differential friction effect of keratin fibres. Journal of Physics D: Applied Physics 23, 406-414.

Chuang, D.T., 1998. Maple syrup urine disease: it has come a long way. Journal of Pedriatrics 132 (3), S17-S23.

Chuang, D.T., Shih, V.E., 1995. Disorders of branched-chain amino acid and keto acid metabolism, in: Scriver, C.R., Beaudet, A.L., Sly, S.W., Valle, D. (Eds.), The Metabolic and Molecular Basis of Inherited Disease, seventh ed. McGraw-Hill, New York, pp. 1239-1277.

Dancis, J., Hutzler, J., Levitz, M., 1960. Metabolism of the white blood cells in maple-syrup-urine disease. Biochimica Biophysica Acta 43, 342-345.

Dancis, J., Hutzler, J., Snyderman, S.E., Cox, R.P., 1972. Enzyme activity in classical and variant forms of maple syrup urine disease. Journal of Pediatrics 81, 312-320.

DiGeorge, A.M., Rezvani, I., Garibaldi, L.R., Schwartz, M., 1982. Prospective study of maple-syrup-urine disease for the first four days of life. New England Journal of Medicine 307, 1492-1495.

Evans, D.J., Lanczki, M., 1997. Cleavage of integral surface lipids of wool by aminolysis. Textile Research Journal 67, 435.

Evans, D.J., Leeder, J.D., Rippon, J.A., Rivett, D.E., 1985. Separation and analysis of the surface lipids of the wool fibre, Proceedings of the Seventh International Wool Textile Research Conference, Tokyo, Japan 1985 p. $135-142$.
Fisher, C.R., Fisher, C.W., Chuang, D.T., Cox, R.P., 1991. Occurrence of a Tyr393 $\rightarrow$ Asn $(Y 393 N)$ mutations in the E1 $\alpha$ gene of the branchedchain a-keto acid dehydrogenase complex in maple syrup urine disease patients from a Mennonite population. American Journal of Human Genetics 49, 429-434.

Jones, L.N., Rivett, D.E., 1997. The role of 18-methyleicosanoic acid in the structure and formation of mammalian hair fibres. Micron 28 (6), $469-485$

Jones, L.N., Peet, D.J., Danks, D.M., Negri, A.P., Rivett, R.E., 1996. Hairs from patients with maple syrup urine disease show a structural defect in the fibre cuticle. Journal of Investigative Dermatology 106 (3), 461-464.

Kao Corporation, EU Patent 0483689 A1, 25th October, 1991.

Martin, A.J.P., 1944. Observations on the theory of felting. Journal of the Society of Dyers and Colourists 60, 325-328.

Matsuda, I., Nebukuni, Y., Mitsubuchi, H., et al., 1990. A T-to-A substitution in the E1 $\alpha$ subunit gene of the branched-chain $\alpha$-ketoacid dehydrogenase complex in two cell lines derived from Mennonite maple syrup urine disease patients. Biochemical and Biophysical Research Communications 172, 646-651.

Menkes, J.H., Hurst, P.L., Craig, J.M., 1954. A new syndro,e: progressive familial infantile cerebral dysfunction associated withh an unusual urinary substance. Pediatrics 14, 462-466.

Mercer, E.H., 1961. Keratin and Keratinisation. Pergamon Press, Oxford.

Silao, C.L.T., Padilla, C.D., Matsuo, M., 2004. A novel deletion creating a new terminal exon of the dihydrolipoyl transacylase gene is a founder mutation of Filipino maple syrup urine disease. Molecular Genetics and Metabolism 81 (2), 100-104.

Smith, J.R., 1998. A quantitative method for analysing AFM images of the outer surfaces of human hair. Journal of Microscopy 191, 223-228.

Smith, J.R., Swift, J.A., 2002. Lamellar subcomponents of the cuticular cell membrane complex of mammalian keratin fibres show friction and hardness contrast. Journal of Microscopy 206, 182-193.

Spurr, A.R., 1969. A low-viscosity epoxy resin embedding medium for electron microscopy. Journal of Ultrastructure Research 26, 31-43.

Swift, J.A., 1991. Fine details on the surface of human hair. International Journal of Cosmetic Science 13 (3), 143-159.

Swift, J.A., 1999. Human hair cuticle: biologically conspired to the owner's advantage. Journal of Cosmetic Science 50, 23-47.

Swift, J.A., Holmes, A.W., 1965. Degradation of human hair by papain. Part III. Some electron microscope observations. Textile Research Journal 35, 1014-1019.

Swift, J.A., Smith, J.R., 2000. Atomic force microscopy of human hair. Scanning 22 (5), 310-318.

Swift, J.A., Smith, J.R., 2001. Microscopic investigations on the epicuticle of mammalian keratin fibres. Journal of Microscopy 204, 203-211.

Wertz, P.W., Downing, D.T., 1988. Integral lipids of human-hair. Lipids 23, 878-881.

Wertz, P.W., Downing, D.T., 1989. Integral lipids of mammalian hair. Comparative Biochemistry and Physiology 92B, 759-761. 